\title{
Associations among the plasma amino acid profile, obesity, and glucose metabolism in Japanese adults with normal glucose tolerance
}

Chisa Takashina ${ }^{1}$, Ichizo Tsujino ${ }^{1 *}$, Taku Watanabe ${ }^{1}$, Shinji Sakaue ${ }^{2}$, Daisuke Ikeda ${ }^{1}$, Asuka Yamada ${ }^{1}$, Takahiro Sato ${ }^{1}$ Hiroshi Ohira ${ }^{2}$, Yoshinori Otsuka ${ }^{3}$, Noriko Oyama-Manabe ${ }^{4}$, Yoichi M. Ito ${ }^{5}$ and Masaharu Nishimura ${ }^{2}$

\begin{abstract}
Background: Amino acids (AAs) are emerging as a new class of effective molecules in the etiology of obesity and diabetes mellitus. However, most investigations have focused on subjects with obesity and/or impaired glucose regulation; the possible involvement of AAs in the initial phase of glucose dysregulation remains poorly understood. Furthermore, little attention has been given to possible associations between the pattern/degree of fat deposition and the plasma AA profile. Our objective was therefore to determine the relationships between plasma AA concentrations and the type/degree of obesity and glucose regulation in Japanese adults with normal glucose tolerance.
\end{abstract}

Methods: Eighty-three subjects with normal glucose tolerance were classified as obese or nonobese and as visceral obesity or nonvisceral obesity. Correlations between the plasma levels of 23 AAs and somatometric measurements, visceral fat area (VFA), subcutaneous fat area (SFA), and 75-g oral glucose tolerance test results were analyzed.

Results: Obesity or visceral obesity was associated with higher levels of branched-chain AAs (isoleucine, leucine, and valine), lysine, tryptophan, cystine, and glutamate but lower levels of asparagine, citrulline, glutamine, glycine, and serine $(p<0.04)$. Age- and gender-adjusted analyses indicated that VFA was positively correlated with tryptophan and glutamate levels, whereas VFA and SFA were negatively correlated with citrulline, glutamine, and glycine levels $(p<$ 0.05). The fasting and 2-h plasma glucose levels or the homeostasis model assessment of insulin resistance were positively correlated with valine, glutamate, and tyrosine levels but negatively correlated with citrulline, glutamine, and glycine levels. The homeostasis model assessment for the $\beta$-cell function index was positively correlated with leucine, tryptophan, valine, and glutamate levels but negatively correlated with citrulline, glutamine, glycine, and serine levels $(p<0.05)$.

Conclusions: The present study identified specific associations between 10 AAs and the type/degree of obesity, and indices of glucose/insulin regulation, in Japanese adults with preserved glucose metabolism. With the growing concern about the increasing prevalence of obesity and diabetes, the possible roles of these AAs as early markers and/or precursors warrant further investigation.

Keywords: Visceral obesity, Oral glucose tolerance test, Insulin secretion, Insulin resistance, Branched-chain amino acid, Glycine, Glutamate, Glutamine

\footnotetext{
* Correspondence: itsujino@med.hokudai.ac.jp

${ }^{1}$ First Department of Medicine, Hokkaido University Hospital, North 14, West

5, Kita-ku, Sapporo 060-8648, Japan

Full list of author information is available at the end of the article
} 


\section{Background}

Globally, the prevalence of diabetes mellitus (DM), impaired glucose tolerance (IGT), and metabolic syndrome is increasing; these disorders are becoming a major economic and social burden [1]. Insulin resistance plays a central role in the pathogenesis of these disorders, through defects in insulin-mediated glucose uptake and/or glucose release by the liver, muscles, and other peripheral tissues. There is a wellestablished epidemiological relationship between obesity and insulin resistance. However, this relationship has been investigated less thoroughly in nondiabetic subjects with normal glucose metabolism than in subjects with DM or IGT.

Amino acids (AAs) are emerging as a new class of effective molecules in the etiology of obesity and DM. During a 12-year follow-up study, plasma levels of branched-chain AAs (BCAAs; isoleucine, leucine, and valine), tyrosine, and phenylalanine were reported to predict the development of DM in nondiabetic subjects [2]. Prior clinical studies have also identified significant associations between the plasma levels of specific AAs and body mass index (BMI) [3, 4] or glucose regulation [5]. However, most investigations involved subjects with obesity and/or impaired glucose regulation [6-8]. Thus, the possible involvement of AAs in the initial phase of glucose dysregulation remains poorly understood. Furthermore, little attention has been given to possible associations between the pattern and degree of fat deposition and the plasma AA profile. This is of clinical interest because visceral adipose tissue demonstrates a stronger association with metabolic disturbances and cardiovascular risks than subcutaneous adipose tissue [9, 10]. Finally, most related studies involved mainly non-Asian subjects $[4,5,11]$, who differ physically and metabolically from the Asian population [12, 13]. Indeed, a community-based observational study documented that Asians have a lower BMI but a higher percentage of body fat than Caucasians [14]. Based on these and other publications from around the world [15, 16], a definition for obesity specific to the Japanese population was proposed as BMI $\geq 25 \mathrm{~kg} / \mathrm{m}^{2}$ [17], which differed from that for Europeans $\left(B M I \geq 30 \mathrm{~kg} / \mathrm{m}^{2}\right.$ ).

The aim of the present study was to investigate the relationships between the plasma AA profile and the type/degree of obesity as well as glucose and insulin regulation, in normoglycemic Japanese adults. Using a detailed analysis of the indices of insulin resistance, sensitivity, and secretion derived from the 75-g oral glucose tolerance test (75-g OGTT), this study identified significant associations between AAs and the development of aberrant glucose regulation in nondiabetic Japanese adults.

\section{Methods}

\section{Subjects}

Ninety-four healthy Japanese volunteers aged 20-60 years were recruited between March 2009 and March 2010 from the medical students, interns, employees, and paramedical staff of Hokkaido University Hospital. We excluded subjects with a history of DM, chronic kidney disease, or liver disease, and those taking medications that could affect glucose metabolism, such as angiotensin-converting enzyme inhibitors, angiotensin II receptor inhibitors, statins, quinolones, antimicrobial agents, and any drug that presents hormonal effects. Finally, those unable to undergo magnetic resonance imaging (MRI) for any reason were excluded. This study was approved by the Ethical Committee on Human Experiments of Hokkaido University Graduate School of Medicine (MED08-003), and written informed consent was obtained from all subjects.

All subjects underwent somatometry, blood sampling, 75-g OGTT, and MRI within a week of their enrollment, during which there was no significant change in their lifestyle, including their dietary intake, or their physical condition.

\section{Somatometry}

Height and body weight (BW) were measured using standard methods on the same day as the blood sampling for plasma AA measurements and the 75-g OGTT. Waist circumference (WC) was measured in the standing position, at the umbilical level, after expiration during natural breathing. BMI was calculated using the following formula: BW $(\mathrm{kg}) /(\text { height }(\mathrm{m}))^{2}$. The subjects were divided into two groups based on the BMI cutoff value for obesity determined for the Japanese population $\left(\mathrm{BMI} \geq 25 \mathrm{~kg} / \mathrm{m}^{2}\right)$ [17].

\section{The 75-g OGTT}

The subjects were tested using the standard 75-g OGTT procedure [18]. Briefly, the subjects were fasted from $11 \mathrm{pm}$ on the previous day until 9 am, when they were given $300 \mathrm{ml}$ of aqueous solution containing $75 \mathrm{~g}$ of glucose to drink within $5 \mathrm{~min}$. Blood samples were collected from an antecubital vein immediately before ingestion of the solution (fasting plasma glucose [FPG]) and 30, 60, 90, and $120 \mathrm{~min}$ after ingestion. The samples were collected in tubes containing fluoride and maintained at $4{ }^{\circ} \mathrm{C}$ until they were centrifuged, within $2 \mathrm{~h}$, to measure the plasma glucose (PG) and plasma insulin levels. The results were interpreted according to the World Health Organization definitions: normal glucose tolerance (NGT; FPG $<110 \mathrm{mg} / \mathrm{dl}$ and 2-h PG $<140 \mathrm{mg} /$ $\mathrm{dl}$ ), impaired fasting glucose (FPG 110-125 mg/dl and 2-h PG $<140 \mathrm{mg} / \mathrm{dl}$ ), IGT (FPG < $126 \mathrm{mg} / \mathrm{dl}$ and 2-h PG 140-199 mg/dl), and DM (FPG $\geq 126 \mathrm{mg} / \mathrm{dl}$ or $2-\mathrm{h}$ PG $\geq$ 
$200 \mathrm{mg} / \mathrm{dl}$ ) [19]. Insulin resistance was determined using the homeostasis model assessment of insulin resistance (HOMA-IR) index, calculated as follows: FPG $(\mathrm{mg} / \mathrm{dl}) \times$ fasting plasma insulin $(\mu \mathrm{U} / \mathrm{ml}) / 405$ [20]. Insulin sensitivity was assessed by calculating the oral glucose insulin sensitivity (OGIS) index using a spreadsheet downloaded from the OGIS website [21, 22]. Insulin secretion was measured using the homeostasis model assessment of $\beta$ cell function index (HOMA- $\beta$ ), calculated as follows: (fasting plasma insulin $(\mu \mathrm{U} / \mathrm{ml}) \times 360) /(\mathrm{FPG}(\mathrm{mg} / \mathrm{dl})-63)$ [20]. Insulin secretion was also expressed in terms of the insulinogenic index (II): 0-30 min insulin increment $(\mu \mathrm{U} /$ $\mathrm{ml}) / 0-30$ min PG increment (mg/dl) [23].

\section{Measurement of plasma AA concentrations}

Separate blood specimens were collected from the antecubital vein into heparinized tubes at the time the fasting blood specimens were obtained for the 75-g OGTT. The plasma was separated at $4{ }^{\circ} \mathrm{C}$ and stored at $-80{ }^{\circ} \mathrm{C}$ until the measurements were made. The plasma was deproteinized in a final concentration of $80 \%$ acetonitrile, and the plasma AAs were identified and quantified using high-performance liquid chromatography (L-8500; Hitachi, Ltd., Tokyo) after derivatization (SRL, Inc., Tokyo) [24, 25].

Twenty-three compounds were analyzed in each plasma sample to cover the main essential AAs acquired from the diet (histidine, isoleucine, leucine, lysine, methionine, phenylalanine, threonine, tryptophan, and valine) as well as the nonessential AAs (alanine, arginine, asparagine, $\alpha$-aminobutyric acid, citrulline, cystine, glutamate, glutamine, glycine, ornithine, proline, serine, taurine, and tyrosine). Of these, the BCAAs are isoleucine, leucine, and valine.

\section{Evaluation of visceral and subcutaneous fat by MRI}

The MRIs were performed with an Achieva $1.5 \mathrm{~T}$ (Philips Medical System, Best, The Netherlands) equipped with a gantry coil. Coronal, sagittal, and longaxis scout images were obtained, covering the whole body from the cervix to the pelvis. Transverse axial fat imaging was then performed during repeated breathholds, using a T1-turbo field echo (TFE) sequence with spectral attenuated inversion recovery (SPAIR) for water suppression. The acquisition parameters were as follows: field of view, $450 \times 310 \mathrm{~mm}$; (repetition time)/(echo time), 3.6/1.7 ms; acquisition matrix, $128 \times 100$; slice thickness, $6 \mathrm{~mm}$; flip angle, $10^{\circ}$; and TFE factor, 30. For SPAIR, the repetition time was $206 \mathrm{~ms}$, and the inversion time was $80 \mathrm{~ms}$.

The areas of visceral and subcutaneous fat were measured using commercially available software (FatChecker, VOX-BASE; J-MAC system, Sapporo, Japan). In brief, a transaxial image at the level of the umbilicus was selected from the stack of abdominal short-axis images. In this image, fat tissue was distinguished from the other tissues (muscles and bones) based on the minimum and maximum signal intensities of each tissue, calculated using an approximation of the Gaussian distribution of the overall histogram. The boundaries between the subcutaneous fat and visceral fat were automatically traced using the software's tracking operation. The subcutaneous fat and visceral fat were identified as the regions outside and inside of these boundaries, respectively. Each fat region identified with this method was visually confirmed, and the border manually corrected as needed. The subcutaneous fat area (SFA) and visceral fat area (VFA) were calculated from the number of pixels in the histograms and the pixel size. The subjects were divided into two groups based on the widely used VFA cutoff value of $100 \mathrm{~cm}^{2}$ : the visceral obesity group $\left(V F A \geq 100 \mathrm{~cm}^{2}\right)$ and the nonvisceral obesity group $\left(\mathrm{VFA}<100 \mathrm{~cm}^{2}\right)$ [17].

\section{Data analysis}

All values are presented as mean \pm SD. The obese and nonobese groups, and the visceral obesity group and nonvisceral obesity groups, were compared in terms of demographic parameters, somatometric indices, 75-g OGTT results, SFA, VFA, and plasma AA concentrations using chi-square tests or unpaired $t$-tests, as appropriate.

Plasma AA concentrations were also compared between men and women using an unpaired $t$-test.

Associations between plasma AA concentrations and other variables (age, BW, BMI, WC, VFA, SFA, FPG, 2-h PG, HOMA-IR, OGIS, HOMA- $\beta$, and II) were assessed by Pearson's correlation analysis and also by multivariate logistic regression analysis adjusted for age and gender.

The reproducibility of the SFA and VFA measurements was determined using randomly selected MRI images from 10 subjects, measured by two investigators (AY and CT) separated by an interval of at least 2 weeks. The reproducibility analysis was conducted using Bland-Altman plots and intraclass correlation coefficients. The investigators were unaware of the somatometric data or the blood test results.

All statistical analyses were conducted using JMP software version 10 , and $p$ values $<0.05$ were considered statistically significant.

\section{Results}

Among the 94 adult volunteers, 11 subjects were excluded from the analysis because the 75-g OGTT test indicated that they had DM $(n=3)$ or IGT $(n=8)$. The remaining 83 subjects, 66 men and 17 women all with NGT, were divided into two groups based on their BMI: obese $(n=10)$ or nonobese $(n=73)$. These subjects were 
also divided into two groups based on their VFA: visceral obesity $(n=18)$ or nonvisceral obesity $(n=65)$. The two pairs of groups were compared in terms of demographic, somatometric, 75-g OGTT, and MRI data (Table 1). The obese group presented significantly higher somatometric parameters (BW, BMI, and WC), FPG, insulin resistance (HOMA-IR), insulin secretion (II), and SFA, but a lower insulin sensitivity (OGIS) than the nonobese group (all $p<0.001)$. The visceral obesity group included significantly more men than the nonvisceral obesity group, as well as significantly higher age, somatometric parameters (BW, BMI, and WC), and II (all $p \leq 0.017$ ).

Table 2 shows that, for the essential AAs, obesity and visceral obesity were both associated with higher plasma concentrations of the three BCAAs (isoleucine, leucine, and valine; all $p \leq 0.033$ ). Lysine levels were higher in the obese group $(p=0.006)$, and tryptophan levels were higher in the visceral obesity group $(p=0.004)$. In contrast, the analysis revealed remarkably different impacts of obesity and visceral obesity on the nonessential AA profile. Obesity was associated with higher levels of cystine and glutamate but lower levels of glycine. Visceral obesity was associated with higher levels of glutamate but lower levels of asparagine, citrulline, glutamine, glycine, and serine.

Comparison of the AA concentrations between men and women showed that those of 11 AAs were higher in men than in women, but serine levels were lower in men than in women (Additional file 1: Table S1). There were significant correlations between age and the plasma concentrations of four AAs (Additional file 2: Table S2). In a subgroup analysis involving only the 66 men, 7 of the 10 AAs that exhibited significantly different concentrations in the overall analysis on 83 subjects still showed different plasma concentrations between visceral and nonvisceral obesity groups (Additional file 3: Table S3). The men were further divided into two subsets (each $n=33$ ) according to whether they were aged above or below the median age of 35. A comparison of AA concentrations showed that significant differences existed for two AAs (glutamate and glutamine) in the older subset ( $>35$ years) and for eight AAs in the younger subset (Additional file 4: Table S4). Overall, these results suggested significant impacts of gender and age on the AA concentrations. At the same time, they also indicated the associations between AA concentrations and visceral obesity because significant differences of the concentrations of some AAs remained between the subjects with or without visceral obesity in the age- and gender-adjusted analyses.

The associations between AAs and the glucose regulation of the NGT subjects was investigated by Pearson's correlation analysis (Table 3, Additional file 5: Table S5) and by age- and gender-adjusted logistic regression analysis (Table 4, Additional file 6: Table S6) of the plasma AA concentrations of all 83 subjects and the

Table 1 Somatometric measurements and the results of 75-g oral glucose tolerance test and magnetic resonance imaging

\begin{tabular}{|c|c|c|c|c|c|c|c|}
\hline & $\begin{array}{l}\text { Overall } \\
(n=83)\end{array}$ & $\begin{array}{l}\text { Obese } \\
(n=10)\end{array}$ & $\begin{array}{l}\text { Nonobese } \\
(n=73)\end{array}$ & $p$ values ${ }^{a}$ & $\begin{array}{l}\text { Visceral obesity } \\
(n=18)\end{array}$ & $\begin{array}{l}\text { Nonvisceral obesity } \\
(n=65)\end{array}$ & $p$ values $^{\mathrm{b}}$ \\
\hline Gender (male/female) & $66 / 17$ & $10 / 0$ & $56 / 17$ & 0.114 & $18 / 0$ & $48 / 17$ & 0.017 \\
\hline Age (years) & $34.0 \pm 5.7$ & $35.2 \pm 3.8$ & $33.8 \pm 5.9$ & 0.487 & $37.1 \pm 7.0$ & $33.2 \pm 5.0$ & 0.009 \\
\hline \multicolumn{8}{|l|}{ Somatometric measurements } \\
\hline Body weight (kg) & $65.5 \pm 9.9$ & $79.5 \pm 8.5$ & $63.5 \pm 8.5$ & $<0.001$ & $72.0 \pm 8.2$ & $63.7 \pm 9.6$ & 0.001 \\
\hline Body mass index $\left(\mathrm{kg} / \mathrm{m}^{2}\right)$ & $22.5 \pm 2.4$ & $26.7 \pm 2.3$ & $21.9 \pm 1.8$ & $<0.001$ & $24.1 \pm 2.6$ & $22.0 \pm 2.2$ & 0.001 \\
\hline Waist circumference (cm) & $78.8 \pm 7.2$ & $88.9 \pm 5.8$ & $77.4 \pm 6.2$ & $<0.001$ & $84.8 \pm 6.7$ & $77.2 \pm 6.4$ & $<0.001$ \\
\hline \multicolumn{8}{|l|}{75 g-oral glucose tolerance test } \\
\hline Fasting plasma glucose (mg/dl) & $91.7 \pm 8.1$ & $100.7 \pm 5.2$ & $90.4 \pm 7.6$ & $<0.001$ & $91.9 \pm 9.4$ & $91.6 \pm 7.7$ & 0.868 \\
\hline 2-h plasma glucose (mg/dl) & $104.3 \pm 16.1$ & $110.3 \pm 17.2$ & $103.4 \pm 15.9$ & 0.209 & $108.1 \pm 19.2$ & $103.2 \pm 15.1$ & 0.255 \\
\hline Insulin resistance (HOMA-IR) & $1.2 \pm 0.8$ & $2.1 \pm 1.6$ & $1.1 \pm 0.5$ & $<0.001$ & $1.5 \pm 1.3$ & $1.2 \pm 0.6$ & 0.067 \\
\hline Insulin sensitivity (OGIS) & $446.6 \pm 47.1$ & $394.0 \pm 52.0$ & $453.8 \pm 41.8$ & $<0.001$ & $436.6 \pm 65.4$ & $449.4 \pm 40.9$ & 0.312 \\
\hline Insulin secretion (HOMA- $\beta$ ) & $71.3 \pm 37.8$ & $78.8 \pm 53.6$ & $70.3 \pm 35.4$ & 0.507 & $82.4 \pm 45.7$ & $68.2 \pm 35.0$ & 0.159 \\
\hline Insulin secretion (II) ${ }^{c}$ & $0.3 \pm 0.2$ & $0.5 \pm 0.4$ & $0.3 \pm 0.1$ & $<0.001$ & $0.4 \pm 0.4$ & $0.3 \pm 0.1$ & 0.004 \\
\hline \multicolumn{8}{|c|}{ Magnetic resonance imaging-derived fat area } \\
\hline Visceral fat area $\left(\mathrm{cm}^{2}\right)$ & $77.1 \pm 34.3$ & $88.0 \pm 39.2$ & $75.6 \pm 33.6$ & 0.285 & $127.6 \pm 27.1$ & $63.1 \pm 19.8$ & $<0.001$ \\
\hline Subcutaneous fat area $\left(\mathrm{cm}^{2}\right)$ & $122.9 \pm 42.5$ & $176.8 \pm 51.2$ & $115.5 \pm 35.6$ & $<0.001$ & $136.4 \pm 43.5$ & $119.2 \pm 41.8$ & 0.129 \\
\hline
\end{tabular}

Data are mean \pm SD. obese: $\mathrm{BMI} \geq 25$, nonobese: $\mathrm{BMI}<25$; visceral obesity: visceral fat area $\geq 100 \mathrm{~cm}^{2}$, nonvisceral obesity: visceral fat area $<100 \mathrm{~cm}^{2}$ HOMA- $\beta$, homeostasis model assessment for $\beta$-cell function index; HOMA-IR, homeostasis model assessment for insulin resistance; II, insulinogenic index; OGIS, oral glucose insulin sensitivity. ${ }^{a}$ obese vs nonobese, ${ }^{b}$ Visceral obesity vs Nonvisceral obesity, ${ }^{c} n=81$ (Data set from 2 subjects were excluded due to unphysiological glucose/insulin levels. Bold numbers indicate significant differences between the two groups with $p<0.05$ 
Table 2 Plasma amino acid concentrations in the obese, nonobese, visceral obese, and nonvisceral obese groups

\begin{tabular}{|c|c|c|c|c|c|c|c|}
\hline$(\mathrm{nmol} / \mathrm{ml})$ & $\begin{array}{l}\text { Overall } \\
(n=83)\end{array}$ & $\begin{array}{l}\text { Obese } \\
(n=10)\end{array}$ & $\begin{array}{l}\text { Nonobese } \\
(n=73)\end{array}$ & $p$ values $^{a}$ & $\begin{array}{l}\text { Visceral obesity } \\
(n=18)\end{array}$ & $\begin{array}{l}\text { Nonvisceral obesity } \\
(n=75)\end{array}$ & $\begin{array}{l}p^{\mathrm{b}} \\
\text { values }^{\mathrm{a}}\end{array}$ \\
\hline \multicolumn{8}{|l|}{ Essential AAs } \\
\hline Histidine & $85.42 \pm 9.71$ & $88.02 \pm 9.95$ & $85.06 \pm 9.69$ & 0.370 & $84.83 \pm 7.94$ & $85.58 \pm 10.19$ & 0.774 \\
\hline Isoleucine & $64.07 \pm 12.09$ & $72.94 \pm 12.74$ & $62.86 \pm 11.57$ & 0.013 & $70.99 \pm 10.59$ & $62.16 \pm 11.85$ & 0.005 \\
\hline Leucine & $125.07 \pm 20.71$ & $139.34 \pm 15.73$ & $123.12 \pm 20.62$ & 0.019 & $136.51 \pm 15.93$ & $121.90 \pm 20.85$ & 0.007 \\
\hline Lysine & $187.46 \pm 26.41$ & $208.75 \pm 23.59$ & $184.55 \pm 25.56$ & 0.006 & $186.83 \pm 21.20$ & $187.64 \pm 27.82$ & 0.909 \\
\hline Methionine & $26.51 \pm 3.71$ & $26.69 \pm 3.89$ & $26.48 \pm 3.71$ & 0.871 & $25.81 \pm 3.97$ & $26.70 \pm 3.65$ & 0.370 \\
\hline Phenylalanine & $57.67 \pm 6.79$ & $59.54 \pm 4.86$ & $57.41 \pm 7.00$ & 0.356 & $59.13 \pm 8.34$ & $57.26 \pm 6.31$ & 0.305 \\
\hline Threonine & $129.23 \pm 26.66$ & $126.43 \pm 19.06$ & $129.61 \pm 27.62$ & 0.726 & $122.77 \pm 23.68$ & $131.02 \pm 27.33$ & 0.248 \\
\hline Tryptophan & $56.04 \pm 10.08$ & $53.93 \pm 6.27$ & $56.33 \pm 10.49$ & 0.484 & $61.94 \pm 15.43$ & $54.40 \pm 7.38$ & 0.004 \\
\hline Valine & $231.17 \pm 30.90$ & $261.89 \pm 27.62$ & $226.96 \pm 29.03$ & $<0.001$ & $244.87 \pm 25.78$ & $227.38 \pm 31.30$ & 0.033 \\
\hline \multicolumn{8}{|l|}{ Nonessential AAs } \\
\hline Alanine & $342.61 \pm 79.73$ & $359.03 \pm 99.02$ & $340.36 \pm 77.28$ & 0.491 & $349.52 \pm 83.12$ & $340.70 \pm 79.33$ & 0.681 \\
\hline Arginine & $66.10 \pm 16.33$ & $70.04 \pm 17.12$ & $65.56 \pm 16.27$ & 0.420 & $64.26 \pm 10.86$ & $66.62 \pm 17.58$ & 0.591 \\
\hline Asparagine & $46.49 \pm 6.19$ & $46.17 \pm 7.03$ & $46.53 \pm 6.12$ & 0.865 & $42.73 \pm 5.92$ & $47.53 \pm 5.89$ & 0.003 \\
\hline$a-A B A$ & $20.11 \pm 5.50$ & $22.01 \pm 5.98$ & $19.85 \pm 5.42$ & 0.247 & $20.17 \pm 6.39$ & $20.10 \pm 5.28$ & 0.963 \\
\hline Citrulline & $28.34 \pm 6.20$ & $26.23 \pm 6.23$ & $28.63 \pm 6.19$ & 0.254 & $25.57 \pm 4.99$ & $29.11 \pm 6.32$ & 0.031 \\
\hline Cystine & $36.67 \pm 6.41$ & $41.47 \pm 7.06$ & $36.02 \pm 6.08$ & 0.011 & $37.59 \pm 7.53$ & $36.42 \pm 6.11$ & 0.497 \\
\hline Glutamate & $57.93 \pm 19.19$ & $70.06 \pm 17.04$ & $56.27 \pm 18.97$ & 0.032 & $71.88 \pm 24.25$ & $54.07 \pm 15.68$ & $<0.001$ \\
\hline Glutamine & $524.27 \pm 55.46$ & $514.17 \pm 80.48$ & $525.66 \pm 51.75$ & 0.542 & $495.84 \pm 60.17$ & $532.15 \pm 51.86$ & 0.013 \\
\hline Glycine & $220.56 \pm 34.87$ & $197.88 \pm 41.43$ & $223.67 \pm 33.00$ & 0.027 & $193.69 \pm 30.16$ & $228.00 \pm 32.51$ & $<0.001$ \\
\hline Ornithine & $80.34 \pm 16.80$ & $86.87 \pm 14.91$ & $79.44 \pm 16.94$ & 0.192 & $81.85 \pm 15.20$ & $79.92 \pm 17.30$ & 0.669 \\
\hline Proline & $147.67 \pm 39.39$ & $141.67 \pm 32.09$ & $148.50 \pm 40.40$ & 0.610 & $151.29 \pm 33.02$ & $146.67 \pm 41.15$ & 0.662 \\
\hline Serine & $116.31 \pm 19.30$ & $112.34 \pm 15.82$ & $116.86 \pm 19.76$ & 0.491 & $104.54 \pm 16.36$ & $119.57 \pm 18.87$ & 0.003 \\
\hline Taurine & $74.99 \pm 18.72$ & $69.79 \pm 15.03$ & $75.70 \pm 19.15$ & 0.352 & $71.52 \pm 15.75$ & $75.95 \pm 19.47$ & 0.377 \\
\hline Tyrosine & $61.26 \pm 9.71$ & $66.44 \pm 13.47$ & $60.55 \pm 8.97$ & 0.072 & $62.76 \pm 9.47$ & $60.84 \pm 9.80$ & 0.461 \\
\hline
\end{tabular}

Data are mean \pm SD. obese: $\mathrm{BMI} \geq 25$, nonobese: $\mathrm{BMI}<25$; visceral obesity: visceral fat area $\geq 100 \mathrm{~cm}^{2}$, nonvisceral obesity: visceral fat area $<100 \mathrm{~cm}^{2}$ $A A$ amino acid, $a-A B A$ a-aminobutyric acid, $B M I$ body mass index

${ }^{a}$ Obese vs Nonobese, ${ }^{b}$ Visceral obesity vs Nonvisceral obesity. Bold numbers indicate significant differences between the two groups with $\mathrm{p}<0.05$

somatometric parameters (BW, BMI, and WC), body fat areas (VFA and SFA), and the indices derived from the 75-g OGTT (HOMA-IR, OGIS, HOMA- $\beta$, and II).

Regarding the essential AAs, the age- and genderadjusted analysis showed that the levels of tryptophan and valine correlated positively with the somatometric parameters (BW, BMI, and WC), VFA, and/or SFA. Leucine, tryptophan, and valine levels exhibited positive correlations with insulin resistance (HOMA-IR) or insulin secretion (HOMA- $\beta$ and II). Notably, none of the $75 \mathrm{~g}$ OGTT parameters were associated with the concentrations of the remaining six essential AAs.

The plasma concentrations of nonessential AAs exhibited positive or negative correlations with the other parameters. The most widespread associations were identified for glutamate and glutamine. Plasma glutamate levels positively correlated with all the somatometric parameters, BW, BMI, WC, VFA and SFA, glucose levels
(FPG and 2 h-PG), insulin resistance (HOMA-IR), and insulin secretion (HOMA- $\beta$ and II) but negatively correlated with insulin sensitivity (OGIS). Interestingly, plasma glutamine levels negatively correlated with all the somatometric parameters and the $75 \mathrm{~g}$-OGTT parameters (except FPG, 2 h-PG, and OGIS). Plasma glycine levels negatively correlated with the somatometric parameters (BW, BMI, and WC), VFA and SFA but correlated positively with insulin sensitivity (OGIS) and negatively with insulin resistance (HOMA-IR) and insulin secretion (HOMA- $\beta$ and II). Citrulline levels negatively correlated with BW, BMI, WC, VFA and SFA, insulin resistance (HOMA-IR), and insulin secretion (HOMA- $\beta$ and II). Conversely, tyrosine levels positively correlated with BW and HOMA-IR but negatively correlated with insulin sensitivity (OGIS). Finally, alanine levels correlated positively with $\mathrm{WC}$ and serine levels correlated negatively with HOMA- $\beta$. 
Table 3 Correlations of plasma amino acid concentrations with indices of obesity and glucose metabolism

\begin{tabular}{|c|c|c|c|c|c|c|c|c|c|c|c|}
\hline & Body weight & $\mathrm{BMl}$ & WC & VFA & SFA & FPG & 2-h PG & HOMA-IR & OGIS & HOMA- $\beta$ & $\|$ \\
\hline \multicolumn{12}{|l|}{ Essential AAs } \\
\hline Histidine & - & - & - & - & - & - & - & - & - & - & - \\
\hline Isoleucine & 0.413 & 0.305 & 0.345 & 0.332 & - & - & - & - & - & - & - \\
\hline Leucine & 0.488 & 0.300 & 0.361 & 0.413 & - & - & - & - & - & - & - \\
\hline Lysine & 0.307 & - & 0.290 & - & - & 0.236 & - & - & -0.257 & - & - \\
\hline Methionine & - & - & - & - & - & - & - & - & - & - & 一 \\
\hline Phenylalanine & 0.276 & - & - & - & - & - & - & - & - & - & - \\
\hline Threonine & - & - & - & - & - & - & - & - & - & - & - \\
\hline Tryptophan & - & - & - & 0.289 & - & - & - & - & - & - & - \\
\hline Valine & 0.539 & 0.387 & 0.482 & 0.397 & - & 0.254 & - & 0.219 & -0.297 & - & - \\
\hline Nonessential AAs & & & & & & & & & & & - \\
\hline Alanine & - & - & - & - & - & - & - & - & - & - & - \\
\hline Arginine & - & - & - & - & - & - & - & - & - & - & - \\
\hline Asparagine & - & - & - & - & - & - & - & - & - & - & - \\
\hline$a-A B A$ & - & - & - & - & - & - & - & - & - & - & - \\
\hline Citrulline & - & - & -0.242 & - & -0.395 & - & - & -0.291 & - & -0.375 & - \\
\hline Cystine & 0.276 & - & 0.285 & - & - & 0.236 & - & - & - & - & - \\
\hline Glutamate & 0.548 & 0.443 & 0.528 & 0.568 & - & 0.439 & 0.302 & 0.292 & -0.458 & - & - \\
\hline Glutamine & -0.241 & -0.289 & -0.326 & -0.272 & -0.396 & - & -0.220 & -0.398 & - & -0.311 & - \\
\hline Glycine & -0.333 & -0.418 & -0.426 & -0.426 & -0.294 & - & -0.247 & -0.317 & 0.303 & -0.231 & - \\
\hline Ornithine & - & - & - & - & - & - & - & - & - & - & - \\
\hline Proline & - & - & - & - & - & - & - & - & - & - & - \\
\hline Serine & -0.368 & -0.273 & -0.351 & -0.379 & - & - & - & - & 0.258 & - & - \\
\hline Taurine & - & - & - & - & - & -0.240 & - & - & - & - & - \\
\hline Tyrosine & 0.335 & - & 0.314 & 0.252 & - & 0.269 & - & - & -0.327 & - & - \\
\hline
\end{tabular}

Each number indicates a correlation coefficient for two variables that showed statistically significant correlation by Pearson's analysis. $A A$ amino acid, $a-A B A$ aaminobutyric acid, BMI body mass index, FPG fasting plasma glucose, HOMA- $\beta$ homeostasis model assessment for $\beta$-cell function index, HOMA-IR homeostasis model assessment of insulin resistance, I/ insulinogenic index, OGIS oral glucose insulin sensitivity, SFA subcutaneous fat area, VFA visceral fat area, WC waist circumference, 2-h PG 2-h plasma glucose

The reproducibility of the fat area measurements performed by two independent observers was validated by using Bland-Altman plots. Only small intraobserver (VFA: $-0.4 \pm 5.9$ (2SD), SFA: $-0.1 \pm 1.9$ ) and interobserver (VFA: $-1.5 \pm 8.4$, SFA: $0.0 \pm 2.0$ ) differences were detected. The intraclass correlation coefficients for intraobserver reproducibility were 1.000 for VFA and SFA, and for interobserver reproducibility, these were 0.9995 for VFA and 1.000 for SFA.

\section{Discussion}

The present study, exclusively involving Japanese adults with NGT, demonstrated that the plasma levels of specific AAs were associated with parameters of obesity, and/or indices of insulin resistance, sensitivity, and secretion derived from the 75-g OGTT. These findings support possible associations of AAs with the type and degree of obesity and glucose regulation in the Asian population with preserved glucose metabolism.
An important result of the present study was the establishment of a link between plasma AA levels and type of obesity: visceral or subcutaneous. We demonstrated that tryptophan is positively associated only with VFA, whereas valine, one of the three BCAAs, is positively associated only with SFA. These data suggest that the known associations between obesity and certain AAs may be derived from a distinct link with visceral fat and/ or subcutaneous fat. A previous study on Japanese adults reported similar results; however, the participants did not undergo 75-g OGTT, and those with DM or IGT were not excluded [26]. Conversely, citrulline, glutamine, and glycine negatively correlated with both VFA and SFA, and glutamate positively correlated with both VFA and SFA. These AAs may have broad associations with the overall deposition of fat, rather than with the pattern of fat deposition. Importantly, $88 \%$ of the subjects were considered nonobese $\left(\mathrm{BMI}<25 \mathrm{~kg} / \mathrm{m}^{2}\right)$, and $78 \%$ of them were considered not to have significant visceral 
Table 4 Age- and gender-adjusted correlations of plasma amino acid concentrations with obesity and glucose metabolism indices

\begin{tabular}{|c|c|c|c|c|c|c|c|c|c|c|c|}
\hline & Body weight & $\mathrm{BMl}$ & WC & VFA & SFA & FPG & 2-hPG & HOMA-IR & OGIS & HOMA- $\beta$ & $\|$ \\
\hline \multicolumn{12}{|l|}{ Essential AAs } \\
\hline Histidine & - & - & - & - & - & - & - & - & - & - & - \\
\hline Isoleucine & - & - & - & - & - & - & - & - & - & - & - \\
\hline Leucine & - & - & - & - & - & - & - & - & - & 0.288 & - \\
\hline Lysine & - & - & - & - & - & - & - & - & - & - & - \\
\hline Methionine & - & - & - & - & - & - & - & - & - & - & - \\
\hline Phenylalanine & - & - & - & - & - & - & - & - & - & - & - \\
\hline Threonine & - & - & - & - & - & - & - & - & - & - & - \\
\hline Tryptophan & - & - & - & 0.206 & - & - & - & - & - & 0.259 & - \\
\hline Valine & 0.202 & 0.247 & 0.296 & - & 0.348 & - & - & 0.380 & - & 0.272 & 0.419 \\
\hline \multicolumn{12}{|l|}{ Nonessential AAs } \\
\hline Alanine & - & - & 0.184 & - & - & - & - & - & - & - & - \\
\hline Arginine & - & - & - & - & - & - & - & - & - & - & - \\
\hline Asparagine & - & - & - & - & - & - & - & - & - & - & - \\
\hline$a-A B A$ & - & - & - & - & - & - & - & - & - & - & - \\
\hline Citrulline & -0.183 & -0.286 & -0.358 & -0.285 & -0.382 & - & - & -0.296 & - & -0.323 & -0.307 \\
\hline Cystine & - & - & - & - & - & - & - & - & - & - & - \\
\hline Glutamate & 0.247 & 0.327 & 0.352 & 0.362 & 0.401 & 0.395 & 0.310 & 0.436 & -0.377 & 0.243 & 0.403 \\
\hline Glutamine & -0.265 & -0.308 & -0.282 & -0.233 & -0362 & - & - & -0395 & - & -0.341 & -0.434 \\
\hline Glycine & -0.221 & -0.364 & -0.316 & -0.311 & -0.311 & - & - & -0.325 & 0.226 & -0.309 & -0.380 \\
\hline Ornithine & - & - & - & - & - & - & - & - & - & - & - \\
\hline Proline & - & - & - & - & - & - & - & - & - & - & - \\
\hline Serine & - & - & - & - & - & - & - & - & - & -0.280 & - \\
\hline Taurine & - & - & - & - & - & - & - & - & - & - & - \\
\hline Tyrosine & 0.164 & - & - & - & - & - & - & 0.226 & -0.238 & - & - \\
\hline
\end{tabular}

Each number indicates a standardized coefficient for two variables that showed significant association in the age- and gender-adjusted multiple logistic regression analysis. $A A$ amino acid, $\alpha$-ABA a-aminobutyric acid, $B M I$ body mass index, $F P G$ fasting plasma glucose, HOMA- $\beta$ homeostasis model assessment for $\beta$-cell function index, HOMA-IR homeostasis model assessment of insulin resistance, II insulinogenic index, OGIS oral glucose insulin sensitivity, SFA subcutaneous fat area, VFA visceral fat area, WC waist circumference, 2-h PG 2-h plasma glucose

obesity $\left(\mathrm{VFA}<100 \mathrm{~cm}^{2}\right)$. Thus, this study demonstrated a significant link between AAs in the blood and body fat in nonobese and nonviscerally obese adult Asians.

Three possibilities could contribute to an explanation of the association between plasma AA levels and the type/degree of obesity identified in the present study: 1 . plasma AA levels affect the type/degree of obesity; 2 . different obesity types affect plasma AA levels; and 3. there are confounding factors that affect both plasma AA levels and obesity.

The first possibility relates to the appetite-regulating effects of certain AAs. Glutamate is a substrate in the formation of gamma aminobutyric acid, a known appetite enhancer [27]. In contrast, glutamine increases the secretion of glucagon-like peptide (GLP)-1, which is an appetite suppressor [28]. Therefore, the positive correlation of glutamate and the negative associations of glutamine with the obesity-related indices may be related to the regulation of food intake. In addition, an animal study showed that glycine reduces visceral fat through the oxidization of free fatty acids in adipose tissue [29]. This function is consistent with the negative correlation between high plasma glycine levels and BMI, VFA, and SFA.

The second possibility is that the two types of obesity could affect the AA levels. For instance, a study on rats and human specimens revealed that BCAAs including valine are catabolized in visceral and subcutaneous adipose tissue [30]. Also, a rat model of obesity exhibited enhanced protein degradation and reduced catabolic activities toward BCAAs, which could increase their plasma levels [31]. However, a different catabolism of AAs in visceral and subcutaneous fat tissue has not been clearly demonstrated, indicating the need for further research to support this speculation.

The third possibility is that confounding factors, such as dietary intake or exercise, may affect AA levels and obesity. For example, glutamate is abundant in wheat 
protein, which is the second highest dietary carbohydrate in Japan. In some subjects, excess wheat intake may have caused both an increase in the levels of glutamate and an increase in BMI, VFA, and SFA. Conversely, prolonged submaximal exercise has been reported to reduce plasma leucine concentration and BMI [32]. This finding may be consistent with the significantly lower concentration of leucine in the nonobese group compared with the obese group.

With regard to the link between AAs and the indices of glucose regulation, the present study identified positive correlations of glutamate concentration with FPG and 2-h PG in the Japanese NGT subjects. This finding is partially supported by prior studies in which plasma glutamate, valine, and tyrosine levels were elevated in patients with metabolic syndrome [6] or type 2 DM [33, 34]. These data support the presence of a mechanistic link between an altered plasma AA profile and aberrant glucose regulation, even in subjects with a preserved glucose metabolism. A recent prospective study on 2,422 normoglycemic individuals demonstrated that the plasma levels of five AAs (isoleucine, leucine, valine, tyrosine, and phenylalanine) predicted the development of diabetes during a 12-year follow-up [2]. These results and ours support a possible role for AA metabolism early in the development of DM.

In the present study, the plasma levels of six AAs exhibited relationships with the index of insulin resistance (HOMA-IR) and/or the index of insulin sensitivity (OGIS). Glutamate and tyrosine levels were positively correlated with HOMA-IR and negatively correlated with OGIS, whereas the opposite was the case for glycine. Single associations were also identified for valine, citrulline, and glutamine (with HOMA-IR). This complex network of interactions between AAs and insulin regulation is partially supported by a recent study conducted on Japanese type 2 DM patients, in which the levels of the BCAAs (isoleucine, leucine, and valine), alanine, glutamate, proline, and tyrosine positively correlated with HOMA-IR [8]. The fact that a positive correlation of valine, glutamate, and tyrosine with HOMA-IR was already present in our NGT subjects allows a speculation that these AAs may be associated with the initial phase of the development of glucose dysregulation. Oral glutamate supplements have been reported to impair insulin sensitivity and to induce the development of diabetes in animal studies [35]. In addition, feeding animals with BCAAs induced insulin resistance, along with the chronic phosphorylation of mTOR, JNK, and IRS1 $1_{\text {Ser307 }}$ [3]. Also, valine is a glycogenic AA and, in a rat study, its oral administration increased the PG level [36]. These data support an incremental effect of glutamate and valine on blood glucose levels. Finally, as tyrosine is a precursor of catecholamines, the accumulation of this AA could promote an increase in blood glucose level through the anti-insulin action of catecholamines. Collectively, prior studies and ours suggest that specific AAs may indirectly induce an increase in blood glucose level through the stimulation of insulin resistance.

Glutamine has been reported to induce the secretion of GLP-1 in healthy and diabetic subjects [28], suggesting that this AA reduces PG levels through the stimulatory effect of GLP-1 on insulin secretion. Also, leucine synergistically stimulates insulin secretion and lowers blood glucose when ingested with glucose in healthy humans [37]. In addition, in healthy first-degree relatives of type 2 DM patients, oral glycine has been reported to increase insulin secretion without affecting insulin sensitivity [11]. Also, in another human study, single oral administration of glycine significantly stimulated insulin secretion whereas, when given together with glucose, it augmented insulin action and attenuated the increase of blood glucose levels [38]. Thus, further studies are required to clarify the mechanisms by which AAs affect glucose/insulin regulation.

WC is a basic and widely used somatometric index of visceral fat adiposity. In Japan, the cutoff levels for visceral fat obesity are $85 \mathrm{~cm}$ for men and $90 \mathrm{~cm}$ for women, because these cutoff levels correlate well with a VFA of $100 \mathrm{~cm}^{2}$ [12]. Indeed, in our NGT subjects, WC was greater in the visceral obesity group than in the nonvisceral obesity group. The correlation analysis indicated that WC was correlated with the plasma levels of six AAs, which is a greater number than for BMI (five AAs) and VFA (five AAs). These suggest that WC is not only a marker of visceral obesity but also a noninvasive parameter that could be used in future studies on possible associations between AAs and obesity and/or glucose regulation.

Our study had some limitations. First, we did not collect lifestyle data for subjects; thus, we could not exclude the possibility that factors related to lifestyle, such as dietary intake and/or physical activity, affected the subjects' plasma AA profiles, obesity, and glucose metabolism [39]. Second, only one participant had a BMI $\geq$ $30 \mathrm{~kg} / \mathrm{m}^{2}$, and so the findings cannot be extrapolated to a population with higher BMI. Similarly, due to the racial variation in the relation between AAs and insulin resistance/sensitivity, the results need to be interpreted cautiously when extrapolated to other races [40]. Finally, the observational nature of the present study did not allow a direct investigation of the causal relationships between the parameters. However, only Japanese adults with preserved glucose metabolism were included in the study following careful selection using the 75-g OGTT. Thus, the results obtained are meaningful for future basic and clinical research on the potential role of AAs 
in the pathogenesis and/or prevention of obesity and DM.

\section{Conclusions}

The present study identified specific associations between 10 AAs and the type and degree of obesity, or indices of glucose/insulin regulation, in Japanese adults with preserved glucose metabolism. With the growing concern about the increasing prevalence of obesity and $\mathrm{DM}$, the possible roles of these AAs as early markers and/or precursors of these conditions warrant further investigation.

\section{Ethics approval and consent to participate}

The present study was approved by the Ethical Committee on Human Experiments of Hokkaido University Graduate School of Medicine (MED08-003), and written informed consent was obtained from all subjects.

\section{Consent for publication \\ Not applicable.}

\section{Additional files}

Additional file 1: Table S1. Comparison of plasma amino acid concentrations between men and women. (DOC $45 \mathrm{~kb}$ )

Additional file 2: Table S2. Correlations between plasma amino acid concentrations and age. (DOC $42 \mathrm{~kb}$ )

Additional file 3: Table S3. Plasma amino acid concentrations in visceral obesity and nonvisceral obesity groups in 66 men. (DOC 46 kb)

Additional file 4: Table S4. Plasma amino acid concentrations in visceral obesity and nonvisceral obesity groups in two age groups. (DOC $56 \mathrm{~kb}$ )

Additional file 5: Table S5. Correlations between plasma amino acid concentration and indices of obesity and glucose metabolism. (XLS 19 kb)

Additional file 6: Age- and gender-adjusted correlations between plasma amino acid concentration and indices of obesity and glucose metabolism. (XLS $20 \mathrm{~kb}$ )

\section{Abbreviations}

AA: Amino acid; VFA: Visceral fat area; SFA: Subcutaneous fat area; BCAA: Branched-chain amino acid; DM: Diabetes mellitus; IGT: Impaired glucose tolerance; BMI: Body mass index; OGTT: Oral glucose tolerance test; MRI: Magnetic resonance imaging; BW: Body weight; WC: Waist circumference; PG: Plasma glucose; NGT: Normal glucose tolerance; IFG: Impaired fasting glucose; FPG: Fasting plasma glucose; HOMAIR: Homeostasis model assessment for insulin resistance; OGIS: Oral glucose insulin sensitivity; HOMA- $\beta$ : Homeostasis model assessment of $\beta$-cell function; II: Insulinogenic index; HPLC: High-performance liquid chromatography; SPAIR: Spectral attenuated inversion recovery; GLP-1: Glucagon-like peptide-

\section{Competing interests}

There were no potential conflicts of interest relevant to this article to report.

\section{Authors' contributions}

SS and DI designed the experiments; $C T, D I$, and AY conducted the experiments; CT, TS, DI, and IT analyzed the data; NOM conducted and analyzed the MRI; CT, TW, and IT wrote the original manuscript; YMI provided advice with and reviewed the statistical analysis; $\mathrm{HO}$ and $\mathrm{YO}$ provided academic suggestions, reviewed, and edited the manuscript; and MN was responsible for the final content. All authors read and approved the final manuscript.

\section{Acknowledgments}

The authors thank J-MAC (Sapporo, JAPAN) for its support in the evaluation of visceral and subcutaneous fat area using MRI, and also thank Crimson Interactive Pvt. Ltd. (Tokyo, JAPAN) for scientific English editing throughout the revised manuscript.

\section{Funding}

There was no financial support for this study.

\section{Author details}

${ }^{1}$ First Department of Medicine, Hokkaido University Hospital, North 14, West 5, Kita-ku, Sapporo 060-8648, Japan. 'First Department of Medicine, Hokkaido University Graduate School of Medicine, Sapporo, Japan. ${ }^{3}$ Department of Human Developmental Science, Faculty of Education, Hokkaido University, Sapporo, Japan. ${ }^{4}$ Department of Diagnostic and Interventional Radiology, Hokkaido University Hospital, Sapporo, Japan. ${ }^{5}$ Department of Biostatistics, Hokkaido University Graduate School of Medicine, Sapporo, Japan.

Received: 7 August 2015 Accepted: 22 December 2015

Published online: 19 January 2016

References

1. Wild S, Roglic G, Green A, Sicree R, King H. Global prevalence of diabetes: estimates for the year 2000 and projections for 2030. Diabetes Care. 2004; 27:1047-53

2. Wang TJ, Larson MG, Vasan RS, Cheng S, Rhee EP, McCabe E, et al. Metabolite profiles and the risk of developing diabetes. Nat Med. 2011;17: 448-53.

3. Newgard CB, An J, Bain JR, Muehlbauer MJ, Stevens RD, Lien LF, et al. A branched-chain amino acid-related metabolic signature that differentiates obese and lean humans and contributes to insulin resistance. Cell Metab. 2009;9:311-26

4. Chevalier S, Marliss EB, Morais JA, Lamarche M, Gougeon R. Whole-body protein anabolic response is resistant to the action of insulin in obese women. Am J Clin Nutr. 2005;82:355-65.

5. Schwenk WF, Haymond MW. Decreased uptake of glucose by human forearm during infusion of leucine, isoleucine, or threonine. Diabetes. 1987; 36:199-204.

6. Kamaura M, Nishijima K, Takahashi M, Ando T, Mizushima S, Tochikubo O. Lifestyle modification in metabolic syndrome and associated changes in plasma amino acid profiles. Circ J. 2010;74:2434-40.

7. Mansour A, Mohajeri-Tehrani MR, Qorbani M, Heshmat R, Larijani B, Hosseini $\mathrm{S}$. Effect of glutamine supplementation on cardiovascular risk factors in patients with type 2 diabetes. Nutrition. 2015:31:119-26.

8. Nakamura H, Jinzu H, Nagao K, Noguchi Y, Shimba N, Miyano H, et al. Plasma amino acid profiles are associated with insulin, C-peptide and adiponectin levels in type 2 diabetic patients. Nutr Diabetes. 2014;4:e133.

9. Liu J, Fox CS, Hickson DA, May WD, Hairston KG, Carr JJ, et al. Impact of abdominal visceral and subcutaneous adipose tissue on cardiometabolic risk factors: the Jackson Heart Study. J Clin Endocrinol Metab. 2010;95:5419-26.

10. Yamashita S, Nakamura T, Shimomura I, Nishida M, Yoshida S, Kotani K, et al. Insulin resistance and body fat distribution. Diabetes Care. 1996;19:287-91.

11. Gonzalez-Ortiz M, Medina-Santillan R, Martinez-Abundis E, von Drateln CR. Effect of glycine on insulin secretion and action in healthy first-degree relatives of type 2 diabetes mellitus patients. Horm Metab Res. 2001:33:358-60.

12. Kadowaki T, Sekikawa A, Murata K, Maegawa H, Takamiya T, Okamura T, et al. Japanese men have larger areas of visceral adipose tissue than Caucasian men in the same levels of waist circumference in a population-based study. Int J Obes (Lond). 2006;30:1163-5.

13. Chiu KC, Chuang LM, Yoon C. Comparison of measured and estimated indices of insulin sensitivity and beta cell function: impact of ethnicity on insulin sensitivity and beta cell function in glucose-tolerant and normotensive subjects. J Clin Endocrinol Metab. 2001;86:1620-5.

14. Wang J, Thornton JC, Russell M, Burastero S, Heymsfield S, Pierson Jr RN Asians have lower body mass index (BMI) but higher percent body fat than do whites: comparisons of anthropometric measurements. Am J Clin Nutr. 1994;60:23-8. 
15. Hsu WC, Araneta MR, Kanaya AM, Chiang JL, Fujimoto W. BMl cut points to identify at-risk Asian Americans for type 2 diabetes screening. Diabetes Care. 2015;38:150-8

16. Consultation WHOE. Appropriate body-mass index for Asian populations and its implications for policy and intervention strategies. Lancet. 2004;363: 157-63.

17. Examination Committee of Criteria for 'Obesity Disease' in J, Japan Society for the Study of O. New criteria for 'obesity disease' in Japan. Circ J. 2002;66: 987-92.

18. WHO Expert Committee on Diabetes Mellitus: Diabetes Mellitus. Tech. Rep. Ser., no. 727. Geneva: WHO; 1985

19. WHO. Definition, diagnosis and classification of diabetes mellitus and its complications. Part 1: diagnosis and classification of diabetes mellitus. Geneva: WHO; 1999.

20. Matthews DR, Hosker JP, Rudenski AS, Naylor BA, Treacher DF, Turner RC. Homeostasis model assessment: insulin resistance and beta-cell function from fasting plasma glucose and insulin concentrations in man Diabetologia. 1985;28:412-9.

21. OGIS (Oral Glucose Insulin Sensitivity) [http://webmet.pd.cnr.it/ogis/, Accessed 1 Dec 2015]

22. Mari A, Pacini G, Murphy E, Ludvik B, Nolan JJ. A model-based method for assessing insulin sensitivity from the oral glucose tolerance test. Diabetes Care. 2001;24:539-48.

23. Seltzer HS, Allen EW, Herron Jr AL, Brennan MT. Insulin secretion in response to glycemic stimulus: relation of delayed initial release to carbohydrate intolerance in mild diabetes mellitus. J Clin Invest. 1967;46:323-35.

24. Deyl Z, Hyanek J, Horakova M. Profiling of amino acids in body fluids and tissues by means of liquid chromatography. J Chromatogr. 1986; 379:177-250.

25. Sugino T, Shirai T, Kajimoto Y, Kajimoto O. L-ornithine supplementation attenuates physical fatigue in healthy volunteers by modulating lipid and amino acid metabolism. Nutr Res. 2008;28:738-43.

26. Yamakado M, Tanaka T, Nagao K, Ishizaka Y, Mitushima T, Tani M, et al. Plasma amino acid profile is associated with visceral fat accumulation in obese Japanese subjects. Clin Obes. 2012;2:29-40.

27. Wu Q, Clark MS, Palmiter RD. Deciphering a neuronal circuit that mediates appetite. Nature. 2012;483:594-7.

28. Greenfield JR, Faroogi IS, Keogh JM, Henning E, Habib AM, Blackwood A, et al. Oral glutamine increases circulating glucagon-like peptide 1, glucagon, and insulin concentrations in lean, obese, and type 2 diabetic subjects. Am J Clin Nutr. 2009;89:106-13.

29. El Hafidi M, Perez I, Zamora J, Soto V, Carvajal-Sandoval G, Banos G. Glycine intake decreases plasma free fatty acids, adipose cell size, and blood pressure in sucrose-fed rats. Am J Physiol Regul Integr Comp Physiol. 2004; 287:R1387-1393.

30. Lackey DE, Lynch CJ, Olson KC, Mostaedi R, Ali M, Smith WH, et al Regulation of adipose branched-chain amino acid catabolism enzyme expression and cross-adipose amino acid flux in human obesity. Am J Physiol Endocrinol Metab. 2013;304:E1175-1187.

31. She $P$, Olson KC, Kadota $Y$, Inukai A, Shimomura $Y$, Hoppel $C L$, et al. Leucine and protein metabolism in obese Zucker rats. PLoS One. 2013:8:e59443.

32. Blomstrand $E, E k S$, Newsholme EA. Influence of ingesting a solution of branched-chain amino acids on plasma and muscle concentrations of amino acids during prolonged submaximal exercise. Nutrition. 1996;12:485-90.

33. Fiehn O, Garvey WT, Newman JW, Lok KH, Hoppel CL, Adams SH. Plasma metabolomic profiles reflective of glucose homeostasis in non-diabetic and type 2 diabetic obese African-American women. PLoS One. 2010;5:e15234.

34. Lips MA, Van Klinken JB, van Harmelen V, Dharuri HK, t Hoen PA, Laros JF, et al. Roux-en-Y gastric bypass surgery, but not calorie restriction, reduces plasma branched-chain amino acids in obese women independent of weight loss or the presence of type 2 diabetes. Diabetes Care. 2014;37: 3150-6.

35. Collison KS, Zaidi MZ, Saleh SM, Inglis A, Mondreal R, Makhoul NJ, et al. Effect of trans-fat, fructose and monosodium glutamate feeding on feline weight gain, adiposity, insulin sensitivity, adipokine and lipid profile. Br J Nutr. 2011;106:218-26.

36. Doi M, Yamaoka I, Fukunaga T, Nakayama M. Isoleucine, a potent plasma glucose-lowering amino acid, stimulates glucose uptake in $\mathrm{C}_{2} \mathrm{C} 12$ myotubes. Biochem Biophys Res Commun. 2003;312:1111-7.
37. Kalogeropoulou D, Lafave L, Schweim K, Gannon MC, Nuttall FQ. Leucine, when ingested with glucose, synergistically stimulates insulin secretion and lowers blood glucose. Metabolism. 2008;57:1747-52.

38. Gannon MC, Nuttall JA, Nuttall FQ. The metabolic response to ingested glycine. Am J Clin Nutr. 2002;76:1302-7.

39. Sekhar RV, Patel SG, Guthikonda AP, Reid M, Balasubramanyam A, Taffet GE, et al. Deficient synthesis of glutathione underlies oxidative stress in aging and can be corrected by dietary cysteine and glycine supplementation. Am J Clin Nutr. 2011;94:847-53.

40. Tillin T, Hughes AD, Wang Q, Wurtz P, Ala-Korpela M, Sattar N, et al. Diabetes risk and amino acid profiles: cross-sectional and prospective analyses of ethnicity, amino acids and diabetes in a South Asian and European cohort from the SABRE (Southall And Brent REvisited) Study. Diabetologia. 2015;58:968-79.

\section{Submit your next manuscript to BioMed Central and we will help you at every step:}

- We accept pre-submission inquiries

- Our selector tool helps you to find the most relevant journal

- We provide round the clock customer support

- Convenient online submission

- Thorough peer review

- Inclusion in PubMed and all major indexing services

- Maximum visibility for your research

Submit your manuscript at www.biomedcentral.com/submit 